\title{
TWO POINT BOUNDARY VALUE PROBLEMS FOR NONLINEAR FUNCTIONAL DIFFERENTIAL EQUATIONS
}

\author{
BY \\ PAUL WALTMAN( $\left.{ }^{1}\right)$ AND JAMES S. W. WONG
}

\begin{abstract}
This paper is concerned with the existence of solutions of two point boundary value problems for functional differential equations. Specifically, we consider

$$
y^{\prime}(t)=L\left(t, y_{t}\right)+f\left(t, y_{t}\right), \quad M y_{a}+N y_{b}=\psi,
$$

where $M$ and $N$ are linear operators on $C[0, h]$. Growth conditions are imposed on $f$ to obtain the existence of solutions. This result is then specialized to the case where $L\left(t, y_{t}\right)=A(t) y(t)$, that is, when the reduced linear equation is an ordinary rather than a functional differential equation. Several examples are discussed to illustrate the results.
\end{abstract}

1. Introduction. Let $C_{h}$ denote the Banach space of continuous functions from $[a-h, a]$ into $E^{n}$, the $n$-dimensional Euclidean space, where we take the sup norm in $C_{h}$, i.e. for $\varphi \in C_{h}$

$$
\|\varphi\|=\sup \{|\varphi(\theta)|: \theta \in[a-h, a]\}
$$

and $|\cdot|$ is any convenient norm on $E^{n}$. Let $L(t, \varphi)$ and $f(t, \varphi)$ be continuous mappings from $[a, b] \times C_{h}$ into $E^{n}$ and for each $t \in[a, b]$, let $L(t, \varphi)$ be a bounded linear transformation from $C_{h}$ into $E^{n}$. For a continuous function $f(t)$ defined on $[a, b]$, let $f_{t}$ denote the element in $C_{h}$ defined by $f(\theta)=f(t+\theta-a), \theta \in[a-h, a]$.

We consider the two point boundary value problem

$$
\begin{gathered}
y^{\prime}(t)=L\left(t, y_{t}\right)+f\left(t, y_{t}\right), \quad t \in[a, b], \\
M y_{a}+N y_{b}=\psi, \quad \psi \in C_{h}, \quad b>a+h,
\end{gathered}
$$

where $M$ and $N$ are bounded linear operators on $C_{h}$. The present work is a continuation of an earlier investigation [8] of R. Fennell and the first named author. A linear version of this problem was posed by Cooke [1]. Results on the linear problem may be found in Halanay [10] and in Henry [12]. The earlier work, [8], considers the special case of $(1.1)$ with $L(t, \varphi) \equiv 0$ and, for most part, $M$ and $N$ were

Received by the editors June 5, 1970 and, in revised form, June 3, 1971.

AMS 1969 subject classifications. Primary 3475; Secondary 3436, 3404.

Key words and phrases. Functional differential equations, boundary value problems, periodic solutions, shooting methods, Fredholm alternative.

(1) This research was supported by the National Science Foundation, Grant NSF-GP-8173 at the University of Iowa, and Grant NSF-GP-19617 at the Courant Institute of Mathematical Sciences, New York University. 
restricted to be $n \times n$ matrices. Moreover, the hypotheses on $M$ and $N$ were such as to preclude the important periodic case. Here the problem is treated in the more general, and seemingly more natural case, namely, when the boundary conditions are given in terms of bounded linear operators. Furthermore, the results also apply to periodic boundary conditions, and hence, with periodic forcing, offer a way of demonstrating the existence of periodic solutions of functional differential equations. As in [8], the basic approach is that of a "shooting method", that is, we seek to find the initial function $q \in C_{h}$, such that the corresponding solution of the initial value problem of (1.1) also satisfies the boundary condition (1.2).

First, in $\S 2$, we discuss basic equations and pertinent hypotheses and also develop some results in the linear case. The main results are given in $\$ 3 . \S 4$ is devoted to a discussion of some important special cases when the linear term $L\left(t, y_{t}\right)$ ceases to be functionally dependent, that is, for the case when $f \equiv 0$, equation (1.1) becomes an ordinary differential equation. In $\$ 5$, we describe how some of the assumptions in $\$ 2$ may be relaxed and indicate other results when $f$ satisfies rates of growth other than (2.1). We conclude in $\S 6$ with examples illustrating how our results may be applied to yield the existence of solutions of boundary value problems and show why some of the conditions are imposed.

2. Preliminaries. We first describe the basic hypothesis on the functions $L(t, \varphi)$ and $f(t, \varphi)$. Let $L(t, \varphi)$ be a continuous linear mapping from $C_{h}$ into $E^{n}$ for each $t \in[a, b]$. We assume

$\left(\mathrm{H}_{1}\right)$ There exists a bounded integrable function $l(t)$ such that $|L(t, \varphi)| \leqq l(t)\|\varphi\|$ for $t \in[a, b]$ and $\varphi \in C_{h}$.

Let $f(t, \varphi)$ be a continuous mapping from $[a, b] \times C_{h}$ into $E^{n}$. We assume also

$\left(\mathrm{H}_{2}\right) f(t, \varphi)$ maps closed bounded subsets of $[a, b] \times C_{h}$ into bounded sets in $E^{n}$ and satisfies

$$
\limsup _{\|\varphi\| \rightarrow \infty} \frac{|f(t, \varphi)|}{\|\varphi\|}=0, \quad \text { uniformly in } t \in[a, b] .
$$

In particular, if $f(t, \varphi)$ is bounded then (2.1) is satisfied.

We consider the first order system of functional differential equations of the form

$$
y^{\prime}(t)=L\left(t, y_{t}\right)+f\left(t, y_{t}\right), \quad t \in[a, b],
$$

and the associated linear equation

$$
x^{\prime}(t)=L\left(t, x_{t}\right), \quad t \in[a, b]
$$

subject to the two point boundary conditions:

$$
M y_{a}+N y_{b}=\psi, \quad b>a+h,
$$


where $\psi \in C_{h}$. In order to prove existence of solutions of the two point boundary value problem (1.1) and (1.2), we assume in addition

$\left(\mathrm{H}_{3}\right)$ Solutions of the initial value problem (1.1) with $y_{a}=q \in C_{h}$ exist and are unique.

We note that the existence of local solution to the initial value problem (1.1) with $y_{a}=q$ is guaranteed by the continuity of $L(t, \varphi)$ and $f(t, \varphi)$ as mappings on $[a, b] \times C_{h}$ into $E^{n}$. The extendibility of a local solution to the entire interval $[a, b]$ is guaranteed by $\left(\mathrm{H}_{1}\right)$ and $\left(\mathrm{H}_{2}\right)$, (see, for example, Hale [11, p. 21]).

To facilitate our discussion, use will be made of properties of the linear nonhomogeneous equation

$$
x^{\prime}(t)=L\left(t, x_{t}\right)+g(t)
$$

where $g \in C[a, b]$. For any initial function $q \in C_{h}$, we write $x(q, g)(t)$ as the solution of (2.3) satisfying $x_{a}(q, g)=q$. For each $g \in C[a, b]$ and $q \in C_{h}$, a solution of the initial value problem exists for $t \geqq a-h$, and is unique, see for example [10] or [11]. Such a solution may be represented by

$$
x(q, g)(t)=x(q, 0)(t)+x(0, g)(t),
$$

$t \geqq a-h$. It follows from Gronwall's inequality, see [11, p. 81$]$, that

$$
\left\|x_{t}(q, g)\right\| \leqq\left\{\|q\|+\int_{a}^{t}|g(s)| d s\right\} \exp \left(\int_{a}^{t} l(s) d s\right)
$$

for $t \geqq a$.

An elementary result for the linear equation (2.3) subject to the boundary condition (1.2) provides motivation for the hypothesis we shall make concerning the nonlinear problem (1.1), (1.2). The following obvious lemma gives a necessary and sufficient condition for the linear boundary value problem (2.3), (1.2) to have a solution

LEMMA (2.1). The two point boundary value problem (2.3), (1.2) has a solution if and only if $N x_{b}(0, g) \in \psi+R(M+N X)$ where $R$ denotes the range and $X$ is an operator on $C_{h}$, defined by

$$
X q=x_{b}(q, 0)
$$

which maps $q \in C_{h}$ into the segment at $b$ of the solution of the initial value problem (2.2) with $x_{a}=q$.

Proof. Suppose that $N x_{b}(0, g) \in \psi+R(M+N X)$. To show that the boundary value problem (2.3), (1.2) has a solution, it suffices to establish that the functional equation

$$
M q+N x_{b}(q, g)=\psi, \quad \psi \in C_{h},
$$


has a solution. Using the decomposition formula (2.4), we can write (2.7) as follows

$$
M q+N X q+N x_{b}(0, g)=\psi,
$$

which admits a solution $q$ by hypothesis. Conversely, if the boundary value problem (2.3), (1.2) has a solution $x(t)$ on $[a-h, b]$, then $x_{a}=q$ satisfies (2.7) and (2.8). Consequently, $N x_{b}(0, g) \in \psi+R(M+N X)$ which completes the proof.

In the discussion to follow, we need the following result concerning the operator $X$ defined in (2.6).

LEMMA (2.2). The operator $X$ is a completely continuous linear operator on $C_{h}$.

Proof. For $q_{1}, q_{2} \in C_{h}$ and scalars $\alpha$ and $\beta$, we define $z(t)=\alpha x\left(q_{1}, 0\right)(t)+$ $\beta x\left(q_{2}, 0\right)(t)-x\left(\alpha q_{1}+\beta q_{2}, 0\right)(t)$. It is easily verified by the linearity of $L(t, \varphi)$ that $z(t)$ satisfies (2.2) and the initial condition $z_{a}=0$. By the uniqueness of the solution of the linear equation (2.2), we obtain $z(t) \equiv 0$, proving that $X$ is linear. Since, in case of linear equations, the solutions depend continuously on initial conditions, $X$ is continuous. However, $X$ is linear, therefore it is bounded. To see that $X$ is also compact, let $\left\{q_{n}\right\}$ be a bounded sequence in $C_{h}$, say $\left\|q_{n}\right\| \leqq B, n=1,2,3, \ldots$ By (2.5), we have

$$
\left\|X q_{n}\right\|=\left\|x_{b}\left(q_{n}, 0\right)\right\| \leqq\left\|q_{n}\right\| \exp \left(\int_{a}^{b} l(s) d s\right)
$$

which is uniformly bounded for all $n$. Since $x\left(q_{n}, 0\right)$ is a solution of (2.2) we have

$$
\begin{aligned}
\left\|x_{b}^{\prime}\left(q_{n}, 0\right)\right\| & =\sup _{b-h \leqq t \leqq b}\left|L\left(t, x_{t}\left(q_{n}\right)\right)\right| \leqq \sup _{b-h \leqq t \leqq b} l(t)\left\|x_{t}\left(q_{n}\right)\right\| \\
& \leqq \sup _{b-h \leqq t \leqq b} l(t)\left\|q_{n}\right\| \exp \left(\int_{a}^{t} l(s) d s\right)
\end{aligned}
$$

which is uniformly bounded for all $n$ by $\left(\mathrm{H}_{1}\right)$. It then follows from the ArzelaAscoli Theorem that $\left\{q_{n}\right\}$ contains a convergent subsequence, proving that $X$ is completely continuous.

3. The general problem. The principal result for the general case is

TheOREM (3.1). Assume that $L(t, \varphi)$ and $f(t, \varphi)$ are continuous mappings on $[a, b] \times C_{h}$ into $R^{n}, L(t, \varphi)$ is linear in $\varphi$ for each $t \in[a, b]$ and satisfies $\left(\mathrm{H}_{1}\right)$, and $f(t, \varphi)$ satisfies $\left(\mathrm{H}_{2}\right)$. Suppose also that $\left(\mathrm{H}_{3}\right)$ holds. If the operator $(M+N X)$, where $M, N$ are given in (1.2) and $X$ by (2.6), has a continuous inverse, then the boundary value problem (1.1), (1.2) has at least one solution.

Proof. For each $q \in C_{h}$, denote by $y(t, q)$ the solution of the initial value problem (1.1) with $y_{a}=q$, and $y_{t}(q)$ the corresponding segment at $t$. Define $g(q)(t)=$ $f\left(t, y_{t}(q)\right)$. Then $y(t, q)$ also satisfies the nonhomogeneous linear equation

$$
x^{\prime}(t)=L\left(t, x_{t}\right)+g(q)(t) .
$$


Using (2.4), we can write

$$
y(t, q)=x(q, g)(t)=x(q, 0)(t)+x(0, g(q))(t) .
$$

Define a mapping $T: C_{h} \rightarrow C_{h}$ by

$$
T q=y_{b}(q)=x_{b}(q, 0)+x_{b}(0, g(q)) \text {. }
$$

A solution to the boundary value problem (1.1), (1.2) is determined by an initial condition which is a solution of the functional equation

$$
(M+N T) q=\psi, \quad \psi \in C_{h} .
$$

This may be rewritten as $(M+N X+N(T-X)) q=\psi, \psi \in C_{h}$. Let $\Gamma=(M+N X)^{-1}$ which exists by hypothesis. Equation (3.3) then is equivalent to

$$
(I+\Gamma N(T-X)) q=\Gamma \psi, \quad \psi \in C_{h},
$$

where $I$ denotes the identity. To show the existence of a solution of (3.4) we use the following theorem (Granas [9], Dubrovskii [3]):

Let $B$ be a completely continuous operator mapping a Banach space $X$ into itself. If

$$
\limsup _{\|\varphi\| \rightarrow \infty} \frac{\|B \varphi\|}{\|\varphi\|}<1
$$

then $R(I-B)=X$.

Note that $\Gamma N$ is continuous by hypothesis, so $B$ will be completely continuous if we can show that $T-X$ is. Let $q \in C_{h}$. Consider the solution $y(t, q)$ of the initial value problem (1.1), $y_{a}=q$. By (2.1) and (2.5), we can estimate the function $\left\|y_{t}(q)\right\|$ by

$$
\left\|y_{t}(q)\right\| \leqq L\left(\|q\|+M_{0}+\int_{a}^{t}\left\|y_{s}(q)\right\| d s\right)
$$

where $L=\exp \left\{\int_{a}^{b} l(s) d s\right\}$ and $M_{0}$ is an appropriate constant. Using (3.6) and the Gronwall inequality, we obtain

$$
\left\|y_{t}(q)\right\| \leqq L\left(\|q\|+M_{0}\right) e^{L(b-a)}=K_{1}\|q\|+K_{2} .
$$

Now let $\left\{q_{n}\right\}$ be any bounded sequence in $C_{h}$. We first note that by (2.5), (3.2) and (3.7), we have

$$
\begin{aligned}
\left\|(T-X) q_{n}\right\| & =\left\|x_{b}\left(0, h\left(q_{n}\right)\right)\right\| \leqq L \int_{a}^{b}\left|f\left(s, y_{s}\left(q_{n}\right)\right)\right| d s \\
& \leqq L \int_{a}^{b}\left(M_{0}+\left\|y_{s}\left(q_{n}\right)\right\|\right) d s \leqq L(b-a)\left(M_{0}+K_{1}\left\|q_{n}\right\|+K_{2}\right),
\end{aligned}
$$

which shows that the sequence $\left\{(T-X) q_{n}\right\}$ is uniformly bounded. On the other hand, by (2.1) the sequence of derivatives $\left\{\left[(T-X) q_{n}\right]^{\prime}\right\}$ satisfies

$$
\left\|x_{b}^{\prime}\left(0, g\left(q_{n}\right)\right)\right\| \leqq \sup _{a \leqq t \leqq b}\left\{l(t)\left\|x_{t}\left(0, g\left(q_{n}\right)\right)\right\|+M_{0}+\left\|y_{t}\left(q_{n}\right)\right\|\right\} \text {. }
$$


Note that for $t \in[a, b]$, we have from (2.4), (2.5) and (3.7)

$$
\left\|x_{t}\left(0, g\left(q_{n}\right)\right)\right\| \leqq\left\|y_{t}\left(q_{n}\right)\right\|+\left\|x_{t}\left(q_{n}, 0\right)\right\| \leqq K_{1}\left\|q_{n}\right\|+K_{2}+L\left\|q_{n}\right\| .
$$

Using the above estimate, assumption $\left(\mathrm{H}_{1}\right)$ and (3.7), we obtain from (3.9) that $\left\{\left\|x_{b}^{\prime}\left(0, h\left(q_{n}\right)\right)\right\|\right\}$ is uniformly bounded. Thus, an application of Arzela-Ascoli Theorem will provide the complete continuity of $T-X$ and hence the operator $B$.

Suppose that there exists a sequence of functions $q_{n} \in C_{h},\left\|q_{n}\right\| \rightarrow \infty$ such that

$$
\beta_{n}=\sup _{a \leqq t \leqq b}\left\|y_{t}\left(q_{n}\right)\right\| \uparrow \infty \quad \text { as } n \rightarrow \infty
$$

Let $0<\varepsilon<1 / K_{1}$. By (2.1) there exists a number $r>0$ such that if $\|\varphi\|>r,|f(t, \varphi)|$ $\leqq \varepsilon\|\varphi\|$ for all $t \in[a, b]$. Since for each $s \in[a, b],\left\|y_{s}\left(q_{n}\right)\right\|>r$ or $\left\|y_{s}\left(q_{n}\right)\right\| \leqq r$, we have

$$
\left\|x_{b}\left(0, g\left(q_{n}\right)\right)\right\| \leqq L \int_{a}^{b}\left|f\left(s, y_{s}\left(q_{n}\right)\right)\right| d s \leqq L(b-a) \operatorname{Max}\left(R, \varepsilon \beta_{n}\right),
$$

where $R=\max \{|f(s, \varphi)|: a \leqq s \leqq b,\|\varphi\| \leqq r\}$ and the numbers $\beta_{n}$ are defined by (3.10). From (3.7), we have $\beta_{n} \leqq K_{1}\left\|q_{n}\right\|+K_{2}$, hence

$$
\left\|x_{b}\left(0, g\left(q_{n}\right)\right)\right\| \leqq L(b-a) \operatorname{Max}\left(R, \varepsilon K_{1}\left\|q_{n}\right\|+\varepsilon K_{2}\right) .
$$

Since $\varepsilon$ is arbitrary, this establishes (3.5) for any sequence $q_{n}$ such that the corresponding sequence $\left\{\beta_{n}\right\} \rightarrow \infty$ as $n \rightarrow \infty$. On the other hand, for any sequence $\left\{q_{n}\right\}$ for which the corresponding sequence $\left\{\beta_{n}\right\}$ is bounded, (3.11) implies

$$
\lim _{\left\|q_{n}\right\| \rightarrow \infty} \frac{1}{\left\|q_{n}\right\|}\left\|x_{b}\left(0, g\left(q_{n}\right)\right)\right\|=0
$$

so that (3.5) holds also. This completes the proof of the theorem.

As an immediate consequence of the above result, we obtain the following important corollary:

Corollary (3.2). Assume that the two point boundary value problem (2.2), (1.2) has only the trivial solution. If $M$ has a closed inverse, then the boundary value problem (1.1) and (1.2) always has a solution.

Proof. Since $C_{h}$ is complete under the sup norm, the closed linear operator $M^{-1}$ is also continuous by the closed graph theorem. Note that the boundary condition (1.2) may be rewritten as

$$
I y_{a}+M^{-1} N y_{b}=M^{-1} \psi, \quad \psi \in C_{h},
$$

where $I$ is the identity operator. Since $X$ is completely continuous by Lemma (2.2), so is the operator $M^{-1} N X$. It now follows from the Fredholm alternative that $I+M^{-1} N X$ has continuous inverse (see Dunford and Schwartz [5, p. 515], or Edwards [6, p. 677]) and the result follows. 
Corollary (3.3) (Fennell [7]). Suppose that the mappings $L$ and $f$ in equation (1.1) are, in addition, periodic with period $P=b-a$, i.e. $L(t+P, \varphi)=L(t, \varphi)$ and $f(t+P, \varphi)=f(t, \varphi)$ for all $t \geqq a$ and all $\varphi \in C_{h}$. If the only P-periodic solution of (2.2) is the identically zero solution, then equation (1.1) has a P-periodic solution on $[a, \infty)$.

Proof. Put $M=I, N=-I$ in the boundary conditions and set $\psi=0$.

REMARK 1. We would like to point out that the condition on the invertibility of the operator $M+N X$ is not as severe as it may seem. If one picks an initial function $q \in C_{h}$, then as we noted above the solution $y(t, q)$ satisfies the nonhomogeneous linear equation (3.1) where $g(q)(t)=f(t, y(q))$. However, a necessary and sufficient condition for the boundary value problem (3.1), (1.2) to have a solution is by Lemma (2.1) that $N h(q) \in \psi+R(M+N X)$. Since one expects to have such a condition hold for all $q \in C_{h}, R(M+N X)=C_{h}$ is a reasonable requirement. That $M+N X$ is one-to-one follows from assuming uniqueness of solutions of the linear boundary value problem (2.2), (1.2).

4. Degenerate cases. In this section, we consider equation (1.1) when the linear part is not a functional on $C_{h}$. More specifically, we consider functional differential equations of the form

$$
y^{\prime}(t)=A(t) y(t)+f\left(t, y_{t}\right), \quad t \in[a, b],
$$

where $f$ is a continuous mapping from $[a, b] \times C_{h}$ into $E^{n}$ and $A(t)$ is a $n \times n$ matrix function continuous in $t$. Equation (4.1) will be considered as a special case of equation (3.1). Thus, we also assume that $\left(\mathrm{H}_{2}\right),\left(\mathrm{H}_{3}\right)$ hold throughout this section.

We consider first the case when $A(t) \equiv 0$ which was earlier discussed in [8]. In this case, the operator $X$ defined by (2.6) reduces to

$$
X q=\bar{q}(a),
$$

where $\bar{q}(a)$ denotes the function in $C_{h}$ which has the constant value $q(a)$. We introduce an equivalent norm on $C_{h}$ which is useful in the present analysis. For each $q \in C_{h}$, we define

$$
\|q\|_{a}=|q(a)|+\|q\|
$$

where $\|\cdot\|$ denotes the sup norm. For a linear operator $T$ on $C_{h}$, we define the operator norm with respect to the norm $\|\cdot\|_{a}$ by

$$
\|T\|_{a}=\sup _{\|q\|_{a}=1}\left(\|T q\|_{a}\right)
$$

Except for the additional hypothesis $\left(\mathrm{H}_{3}\right)$ (cf. end of Remark 5), the following result is an extension of the main theorem in [8]:

TheOREM (4.1). If $\Gamma_{0}=(M+N)^{-1}$ exists, is continuous, and $\left\|\Gamma_{0} N\right\|_{a}<1$, then the boundary value problem (4.1), (1.2) with $A(t) \equiv 0$ has a solution. 
Proof. In view of Theorem (3.1), it suffices to show that $(M+N X)^{-1}$ exists and is continuous, where the operator $X$ is given by (4.2). Consider solving the functional equation

$$
(M+N X) q=\varphi, \quad \varphi \in C_{h},
$$

which may be rewritten as

and then as

$$
(M+N+N(X-I)) q=\varphi, \quad \varphi \in C_{h},
$$

$$
\left(I+\Gamma_{0} N(X-I)\right) q=\Gamma_{0} \varphi, \quad \varphi \in C_{h} .
$$

Observe that for $q_{1}, q_{2} \in C_{h}$,

$$
\begin{aligned}
\left\|(X-I) q_{1}-(X-I) q_{2}\right\|_{a} & =\left\|(X-I) q_{1}-(X-I) q_{2}\right\| \\
& \leqq\left\|\bar{q}_{1}(a)-\bar{q}_{2}(a)\right\|+\left\|q_{1}-q_{2}\right\| \\
& =\left|q_{1}(a)-q_{2}(a)\right|+\left\|q_{1}-q_{2}\right\|=\left\|q_{1}-q_{2}\right\|_{a} .
\end{aligned}
$$

Since $\left\|\Gamma_{0} N\right\|_{a}<1$, the operator $\Gamma_{0} N(X-I)$ defines a contraction on $C_{h}$. Thus, equation (4.6) has a unique solution for every $\varphi \in C_{h}$. It is also easy to see that the operator $\left(I+\Gamma_{0} N(X-I)\right)^{-1}$ is Lipschitzian. Therefore

$$
(M+N X)^{-1}=\left(I+\Gamma_{0} N(X-I)\right)^{-1} \Gamma_{0}
$$

is also Lipschitzian and in particular continuous. This completes the proof.

REMARK 2. Theorem (4.1) was first given in [8] where the boundary operators $M, N$ were assumed to be $n \times n$ matrices and $\psi=0$ in the boundary condition (1.2). The proof there explores the dual role played by $n \times n$ matrices as operators from $E^{n}$ into $E^{n}$ and also as operators from $C_{h}$ into $C_{h}$. Suppose that $M, N$ are $n \times n$ matrices; then $\Gamma_{0} N$ is also a $n \times n$ matrix. We note that

$$
\begin{aligned}
\left\|\Gamma_{0} N\right\|_{a} & =\sup _{\|q\|_{a}=1}\left\{\left|\Gamma_{0} N q(a)\right|+\left\|\Gamma_{0} N q\right\|\right\} \\
& =\sup _{\|\|_{a}=1}\left\{\left|\Gamma_{0} N q(a)\right|+\sup _{a-h \leqq \theta \leqq a}\left|\Gamma_{0} N q(\theta)\right|\right\} \\
& \leqq\left|\Gamma_{0} N\right| \sup _{\|q\|_{a}=1}\{|q(a)|+\|q\|\}=\left|\Gamma_{0} N\right|,
\end{aligned}
$$

where $\left|\Gamma_{0} N\right|$ denotes the matrix norm of $\Gamma_{0} N$. Thus, the present condition that $\left\|\Gamma_{0} N\right\|_{a}<1$ is less stringent than that of $\left|\Gamma_{0} N\right|<1$ used in [8].

REMARK 3. We note that the assumption on the existence of $\Gamma_{0}$ rules out the possibility of allowing periodic boundary conditions such as $M=I$ and $N=-I$. This is to be expected, since the reduced equation of (4.1), namely $x^{\prime}=0$, is an ordinary differential equation which for periodic boundary conditions has any constant as a solution.

REMARK 4. Suppose that the $n$-dimensional space $E^{n}$ is equipped with the Euclidean norm. Let $M, N$ be positive definite symmetric matrices. It is easy to see that if $M$ and $N$ commute then $\Gamma_{0}$ exists and satisfies $\left|\Gamma_{0} N\right|<1$. On the other hand, 
the result is not true without the commutativity assumption on $M$ and $N$. Consider the following $2 \times 2$ matrices

$$
M=\left(\begin{array}{cc}
A & A^{-1} \\
A^{-1} & A^{-1}
\end{array}\right), \quad N=\left(\begin{array}{cc}
A & 1-A^{-1} \\
1-A^{-1} & 2 A^{-1}
\end{array}\right),
$$

where $A$ is some large positive number. Clearly, $M, N$ are positive definite if $A>1$ and in fact with positive entries in this case. It is easily found that

and

$$
(M+N)^{-1} M=\frac{1}{5}\left(\begin{array}{cc}
3-A^{-1} & 3 A^{-2}-A^{-1} \\
2-A & 2-A^{-1}
\end{array}\right)
$$

$$
(M+N)^{-1} N=\frac{1}{5}\left(\begin{array}{cc}
2+A^{-1} & A^{-1}-3 A^{-2} \\
A-2 & 3+A^{-1}
\end{array}\right) .
$$

Now consider the vector $x=(1,0)$. We find that $(M+N)^{-1} M x=\frac{1}{5}\left(3-A^{-1}, 2-A\right)$ and $(M+N)^{-1} N x=\frac{1}{5}\left(2+A^{-1}, A-2\right)$ which shows that the operator norms of $(M+N)^{-1} M$ and $(M+N)^{-1} N$ cannot be less than one if $A$ is large.

We now consider equation (4.1) when $A(t) \neq 0$. In view of Theorem (4.1), it is natural to seek conditions on $A(t)$ which will allow periodic boundary conditions. However, since in this case the reduced equation

$$
x^{\prime}(t)=A(t) x(t), \quad t \in[a, b],
$$

is an ordinary differential equation, the mapping $X$ defined by (2.6) will assign the same value to the different functions $q_{1}, q_{2} \in C_{h}$ as long as $q_{1}(a)=q_{2}(a)$. Therefore it will be unreasonable to expect that the operator $M+N X$ be invertible. This difficulty already occurs in Theorem (4.1). There we imposed the contractive condition $\left\|\Gamma_{0} N\right\|_{a}<1$ in order to insure the invertibility of $M+N X$. Here we wish to seek alternative conditions in order not to preclude the periodic boundary conditions. Here, we assume also that $M$ and $N$ are $n \times n$ matrices and wish to obtain an extension of the main result of [8] in another direction. Let $\Phi(t)$ be the fundamental solution matrix of (4.7) satisfying $\Phi(a)=I$, the identity matrix. We define an operator $X_{\odot}: C_{h} \rightarrow C_{h}$ by

$$
\left(X_{\Phi} q\right)(\theta)=\Phi(b-a+\theta) q(\theta), \quad \theta \in[a-h, a] .
$$

We are now ready to state the desired result.

Theorem (4.2). Let $M$ and $N$ be $n \times n$ matrices. Suppose that for all $\theta \in[a-h, a]$ the matrix $M+N \Phi(b-a+\theta)$ is nonsingular and that $\Gamma(\theta)=(M+N \Phi(b-a+\theta))^{-1}$ satisfies

$$
\left\|\Gamma N X_{\Phi}\right\|=\sup _{a-h \leqq \theta \leqq a}|\Gamma(\theta) N \Phi(b-a+\theta)|<1 .
$$

Then the boundary value problem (4.1), (1.2) has at least one solution. 
Proof. The segment $y_{t}$ of a solution $y(t, q)$ of equation (4.1) can be represented as

$$
y_{t}(\theta)=\Phi(t-a+\theta)\left\{q(a)+\int_{a}^{t-a+\theta} \Phi^{-1}(s) f\left(s, y_{s}(q)\right) d s\right\}
$$

for $\theta \in[a-h, a]$. Let $T_{0}$ be a mapping defined on $C_{h}$ by

$$
T_{0} q(\theta)=q(a)+\int_{a}^{b-a+\theta} \Phi^{-1}(s) f\left(s, y_{s}(q)\right) d s .
$$

Using (4.11) and (4.12), we can write $y_{b}(q)=X_{\Phi} T_{0} q$. Thus a solution to the boundary value problem (4.1), (1.2) may be found by solving the functional equation

$$
\left(M+N X_{\Phi} T_{0}\right) q=\psi, \quad \psi \in C_{h} .
$$

Let $\Gamma$ be an operator on $C_{h}$ defined by

$$
(\Gamma q)(\theta)=\Gamma(\theta) q(\theta), \quad \theta \in[a-h, a] .
$$

The operator $\Gamma$ just defined in (4.14) is clearly invertible. Therefore, equation (4.13) is equivalent to

$$
\left(I+\Gamma N X_{\Phi}\left(T_{0}-I\right)\right) q=\Gamma \psi, \quad \psi \in C_{h} .
$$

Decompose the operator $T_{0}-I$ as the sum of two operators $T_{1}$ and $T_{2}$ defined by

$$
\begin{gathered}
T_{1} q(\theta)=q(a)-q(\theta), \\
T_{2} q(\theta)=\int_{a}^{b-a+\theta} \Phi^{-1}(s) f\left(s, y_{s}(q)\right) d s .
\end{gathered}
$$

To obtain a solution of equation (4.15), we invoke a result of Nashed and Wong [14, Theorem 3], and show that the operator $\Gamma N X_{\Phi} T_{1}$ is a contraction and the operator $\Gamma N X_{\Phi} T_{2}$ is completely continuous and is asymptotic to zero in the sense of [3]. As in the proof of Theorem (4.1), we shall work with the new norm introduced in (4.3). We first show that the operator $\Gamma N X_{\Phi} T_{1}$ is a contraction on $C_{h}$ with respect to the new norm \|\|$_{a}$. Let $q_{1}, q_{2} \in C_{h}$, we have from (4.16) that for any $\theta \in[a-h, a]$

$$
\begin{aligned}
\left|\left(T_{1} q_{1}-T_{1} q_{2}\right)(\theta)\right| & \leqq\left|q_{1}(a)-q_{2}(a)\right|+\left|q_{1}(\theta)-q_{2}(\theta)\right| \\
& \leqq\left|q_{1}(a)-q_{2}(a)\right|+\left\|q_{1}-q_{2}\right\|=\left\|q_{1}-q_{2}\right\|_{a},
\end{aligned}
$$

from which it follows that

$$
\left\|T_{1} q_{1}-T_{1} q_{2}\right\|_{a} \leqq\left\|q_{1}-q_{2}\right\|_{a} .
$$

Now condition (4.10) together with (4.18) show that $\Gamma N X_{\Phi} T_{1}$ is a contraction. The fact that $\Gamma N X_{\Phi} T_{2}$ is completely continuous follows the same way as in the proof of Theorem (3.1). Here the operator $T_{2}$ defined by (4.17) is completely continuous, since $f$ satisfies $\left(\mathrm{H}_{2}\right)$. Using (4.11), we can obtain the estimate on $y_{t}(q)$ :

$$
\left\|y_{t}(q)\right\| \leqq K\left(|q(a)|+\int_{a}^{t}\left|\Phi^{-1}(s) f\left(s, y_{s}(q)\right)\right| d s\right),
$$


where $K=\sup _{a-h \leqq t \leqq b}|\Phi(t)|$. Using (2.1) and the Gronwall's inequality, we can deduce from (4.19) the existence of a positive constant $K_{1}$ and $K_{2}$ such that $\left\|y_{t}(q)\right\| \leqq K_{1}\|q\|+K_{2}$ for all $t \in[a, b]$. The last fact can be used to establish that the operator $T_{2}$ is asymptotic to zero, i.e.

$$
\lim _{\|q\|_{a} \rightarrow \infty} \frac{\left\|T_{2} q\right\|_{a}}{\|q\|_{a}}=0
$$

and therefore the operator $\Gamma N X_{\Phi} T_{2}$ is also. This completes the proof.

REMARK 5. The invertibility of the matrix $M+N \Phi(b-a+\theta)$ for each $\theta \in[a-h, a]$ can be guaranteed by the following requirement:

$\left(\mathrm{H}_{4}\right)$ Equation (4.7) subject to the boundary condition $M x(a)+N x(\tau)=0$ has only the trivial solution for each $\tau \in[b-h, b]$.

To see this, we note only that a matrix is invertible if and only if it is one-to-one. Now suppose that for two vectors $v_{1}, v_{2} \in E^{n}$,

$$
(M+N \Phi(b-a+\theta)) v_{1}=(M+N \Phi(b-a+\theta)) v_{2} .
$$

Since $\theta \in[a-h, a]$, we have $b-a+\theta=\tau \in[b-h, b]$. Consider the solution $\Phi(t)$ $\left(v_{1}-v_{2}\right)$ of equation (4.7) which satisfies

$$
M \Phi(a)\left(v_{1}-v_{2}\right)+N \Phi(\tau)\left(v_{1}-v_{2}\right)=(M+N \Phi(\tau)) v_{1}-(M+N \Phi(\tau)) v_{2}=0 .
$$

Therefore, $\left(\mathrm{H}_{4}\right)$ implies that $\Phi(t)\left(v_{1}-v_{2}\right)=0$, or $v_{1}=v_{2}$. In the special case when $A(t) \equiv 0$, assumption $\left(\mathrm{H}_{4}\right)$ is clearly satisfied provided that $M+N$ is invertible. In this case, the fundamental solution matrix $\Phi(t)$ becomes the identity matrix so the requirement (4.10) reduces to $\left|(M+N)^{-1} N\right|<1$. Thus, except for the assumed uniqueness of solutions of initial value problems, namely $\left(\mathrm{H}_{3}\right)$, Theorem 1 of [8] becomes a special case of Theorem (4.2). In fact, since our discussion is limited only to matrices, the approximation technique devised by Kato [13] could be used here in the same way as was done in [8] to remove this restriction. A similar comment applies to Theorem (4.1) if $M$ and $N$ are restricted to be matrix operatcrs.

5. Other results. In this section we consider two alternatives to the hypothesis $\left(\mathrm{H}_{2}\right)$. Both involve making the interval sufficiently small in order to prove the existence of a solution. First, suppose instead of $\left(\mathrm{H}_{2}\right)$ we assurce the weaker hypothesis:

$\left(\mathrm{H}_{2}\right)^{\prime}$ The mapping $f$ is bounded on some sphere, i.e. there exist positive constants $r$ and $K$ such that $\|\varphi\| \leqq r$ implies $|f(t, \varphi)| \leqq K$ for all $t \in[a, b]$.

Denote by $f^{*}(t, \varphi)$ the bounded continuous extension of $f(t, \varphi)$ which is equal to $f(t, \varphi)$ on $\|\varphi\| \leqq r$ and $[a, b]$. The existence of $f^{*}$ is given by a result of Dugundji [4]. We now consider

$$
y^{\prime}(t)=L\left(t, y_{t}\right)+f^{*}\left(t, y_{t}\right)
$$


subject to the boundary condition (1.2) with some prescribed $\psi \in C_{h}$. Let $X$ be the operator on $C_{h}$ defined by (2.6). Suppose that the operator $M+N X$ has a continuous inverse, then Theorem (3.1) establishes the existence of a solution of (5.1) and (1.2). However, since the theorem yields no information about the size of $\|q\|$, the correct initial function, it is not possible to conclude that this solution also solves (1.1) and (1.2). The following result shows that existence to the original problem (1.1), (1.2) is always guaranteed provided that the interval $[a, b]$ is sufficiently small.

TheOREM (5.1). Let $L(t, \varphi)$ and $f(t, \varphi)$ be given as in $\$ 2$ and also assume that $\left(\mathrm{H}_{1}\right),\left(\mathrm{H}_{2}\right)^{\prime},\left(\mathrm{H}_{3}\right)$ hold. If the operator $M+N X$ has a bounded inverse and if $b-a$ is sufficiently small, then the boundary value problem (1.1), (1.2) has a solution for every $\psi \in C_{h}$ such that $\|\Gamma \psi\|<r$.

Proof. We proceed in the same manner as in the proof of Theorem (3.1), i.e. we wish to establish the existence of a solution to the functional equation (3.3) or (3.4). Consider the operator $F$ defined by

$$
F q=\Gamma \psi-\Gamma N(T-X) q, \quad \psi \in C_{h},
$$

where the mappings $\Gamma$ and $T$ are defined by $(M+N X)^{-1}$ and (3.2) respectively. It is clear that a fixed point of the mapping $F$ defined by (5.2) yields a solution to equation (3.3) and hence a solution to the boundary value problem (1.1), (1.2). Let $S_{\alpha}=\left\{q: q \in C_{h},\|q-\Gamma \psi\| \leqq \alpha\right\}$ denote the sphere of radius $\alpha$ centered at $\Gamma \psi$. We show that, for sufficiently small $\alpha$, the mapping $F$ maps $S_{\alpha}$ into $S_{\alpha}$ and is completely continuous with respect to $S_{\alpha}$. The existence of the desired fixed point then follows from the Schauder fixed point theorem. For any $q \in S_{\alpha}$, any $\alpha>0$, we have

$$
\|F q-\Gamma \psi\| \leqq\|\Gamma N\|\left\|x_{b}(0, g(t))\right\|,
$$

where $g(t)=f^{*}\left(t, y_{t}(q)\right)$. As long as we have $\left\|y_{t}(q)\right\| \leqq r, g(t)=f\left(t, y_{t}(q)\right)$. Hence using $\left(\mathrm{H}_{2}\right)^{\prime}$, we find by $(2.5)$

$$
\|F q-\Gamma \psi\| \leqq\|\Gamma N\| K L_{a, b}(b-a),
$$

where $L_{a, b}=\exp \left(\int_{a}^{b} l(s) d s\right)$. On the other hand, in order that $\left\|y_{t}(q)\right\| \leqq r$ it is sufficient that $\{\|q-\Gamma \psi\|+\|\Gamma \psi\|+K(b-a)\} L_{a, b} \leqq r$, or equivalently, taking $\alpha=$ $\|\Gamma N\| K L_{a, b}(b-a)$, we have

$$
\|\Gamma \psi\| L_{a, b}+\left(\|\Gamma N\| L_{a, b}+1\right) K L_{a, b}(b-a) \leqq r .
$$

Note that $L_{a, b}(b-a)$ tends to zero and $L_{a, b}$ tends to 1 as $b-a$ tends to zero. Hence, it is possible to restrict $b-a$ so that (5.4) holds. For such restricted intervals, we define $\alpha$ to be the right-hand side of (5.3). Then we see that $F\left(S_{\alpha}\right) \subseteq S_{\alpha}$ and for all $q \in S_{\alpha}$, the solution $y(t, q)$ of (1.1) coincides with that of (5.1). The operator $T-X$ is completely continuous as already established in Theorem (3.1). Hence, $F$ is also completely continuous, proving the theorem.

We note that if $\psi=0$ in the boundary condition (1.2) then the condition $\|\Gamma \psi\|<r$ imposed in Theorem (5.1) is always satisfied. On the other hand, such a condition 
can be waived if we assume the stronger hypothesis than $\left(\mathrm{H}_{2}\right)^{\prime}$ that $f$ be bounded on every bounded closed subset in $[a, b] \times C_{h}$.

Now we consider the other alternative to hypothesis $\left(\mathrm{H}_{2}\right)$, namely, when $f$ satisfies a uniform Lipschitz condition of the form

$$
\left|f\left(t, \varphi_{1}\right)-f\left(t, \varphi_{2}\right)\right| \leqq K\left\|\varphi_{1}-\varphi_{2}\right\| .
$$

Putting $\varphi_{2}=0$ in (5.5), we obtain

$$
|f(t, \varphi)| \leqq|f(t, 0)|+K\|\varphi\| \leqq \sup _{t \in[a, b]}|f(t, 0)|+K\|\varphi\|,
$$

for all $\varphi \in C_{h}$. In particular, the above discussion applies here also. However, with the Lipschitz condition (5.5), a direct argument is possible using the contraction mapping theorem, and this has the advantage of yielding uniqueness as well. We note that condition (5.5) implies $\left(\mathrm{H}_{3}\right)$.

THEOREM (5.2). Let $L(t, \varphi)$ and $f(t, \varphi)$ be given as in $§ 2$ satisfying $\left(\mathrm{H}_{1}\right)$ and (5.5). If the operator $M+N X$ has a bounded inverse and if $b-a$ is sufficiently small, then the boundary value problem (1.1), (1.2) has a unique solution.

Proof. We proceed in the same way as the proof of Theorem (5.1). Consider the operator $F$ on $C_{h}$ defined by (5.2). To prove the theorem, it suffices to show that the mapping $F$ defines a contraction on $C_{h}$ when the interval $[a, b]$ is made sufficiently small. For $q_{1}, q_{2} \in C_{h}$, we note that

$$
\begin{aligned}
\left\|F q_{1}-F q_{2}\right\| & \leqq\|\Gamma N\|\left\|(T-X) q_{1}-(T-X) q_{2}\right\| \\
& =\|\Gamma N\|\left\|x_{b}\left(0, g\left(q_{1}\right)\right)-x_{b}\left(0, g\left(q_{2}\right)\right)\right\|,
\end{aligned}
$$

where $g(q)(t)=f\left(t, y_{t}(q)\right)$. Observe that from (5.5), we have

$$
\begin{aligned}
\left\|x_{b}\left(0, g\left(q_{1}\right)\right)-x_{b}\left(0, g\left(q_{2}\right)\right)\right\| & \leqq \int_{a}^{b}\left|\Gamma\left(s, y_{s}\left(q_{1}\right)\right)-\Gamma\left(s, y_{s}\left(q_{2}\right)\right)\right| d s \\
& \leqq K \int_{a}^{b}\left\|y_{s}\left(q_{1}\right)-y_{s}\left(q_{2}\right)\right\| d s .
\end{aligned}
$$

It is well known [10] that if $f$ satisfies the Lipschitz condition (5.5) and $L$ satisfies $\left(\mathrm{H}_{1}\right)$ then solutions of (1.1) satisfy

$$
\left\|y_{t}\left(q_{1}\right)-y_{t}\left(q_{2}\right)\right\| \leqq C\left\|q_{1}-q_{2}\right\|, \quad t \in[a, b] .
$$

Using (5.7) and (5.6), we obtain

$$
\left\|F q_{1}-F q_{2}\right\| \leqq\|\Gamma N\| K C(b-a)\left\|q_{1}-q_{2}\right\|,
$$

which clearly defines a contraction with sufficiently small $b-a$.

6. Examples. In this section, we present several examples to illustrate how theorems in previous sections may be used to yield existence of solutions of specific boundary value problems. We also present problems which attempt to isolate some 
of the difficulties encountered in boundary value problems for functional differential equations and which motivate some of the hypothesis imposed in our theorems. The boundary operators involved and the equations discussed are kept simple to avoid lengthy computations.

We first show that it is in fact possible to verify the hypothesis of Theorem 3.1 directly. Consider the functional differential equation

$$
y^{\prime}(t)=y(t-1)+f\left(t, y_{t}\right), \quad t \in[0,2],
$$

subject to the boundary conditions

$$
y_{0}=\frac{1}{4} y_{2}, \quad y_{0}, y_{2} \in C[-1,0] .
$$

Equations (6.1) and (6.2) describe a special case of the boundary value problem (1.1) and (1.2) we considered in $\S 3$. Here $L\left(t, y_{t}\right)=y(t-1), M=I, N=-\frac{1}{4} I, a=0$, $b=2, h=1, \psi=0$ and $f$ is any mapping from $[0,2] \times C[-1,0]$ into $R^{n}$ which satisfies hypothesis $\left(\mathrm{H}_{1}\right)$ and $\left(\mathrm{H}_{2}\right)$, e.g. $f(t, \varphi)=\lambda(t) \varphi^{\sigma}, 0<\sigma<1, \lambda \in C[0,2]$. In this case, the initial value problem for the homogeneous linear equation

$$
x^{\prime}(t)=x(t-1), \quad t \in[0,2],
$$

can be solved by the method of steps, i.e. for any initial function $q \in C[-1,0]$, we have from (6.3)

$$
\begin{aligned}
& x(t)=q(0)+\int_{0}^{t} q(s-1) d s, \quad 0 \leqq t \leqq 1, \\
& =q(0)+\int_{0}^{1} q(s-1) d s+q(0)(t-1) \\
& +\int_{1}^{t} \int_{0}^{u-1} q(s-1) d s d u, \quad 1 \leqq t \leqq 2 .
\end{aligned}
$$

Thus, $X q(\theta)$ is given by the second part of (6.4) (setting $t=2+\theta, \theta \in[-1,0]$ ). It is easy to see that $\sup _{\|q\|=1}\|X q\|<4$, from which it follows that the operator $\left(I-\frac{1}{4} X\right)$ has a continuous inverse. Hence, Theorem (3.1) yields the existence of a solution to the boundary value problem $(6.1),(6.2)$.

We modify the above example to show how Corollary (3.3) may be applied to obtain the existence of a periodic solution. Consider

$$
y^{\prime}(t)=y(t-1)+f\left(y_{t}\right) \sin t, \quad t \in R,
$$

together with the boundary condition

$$
y_{0}=y_{2 \pi}, \quad y_{0}, y_{2 \pi} \in C[-2 \pi, 0] .
$$

We assume that $f\left(y_{t}\right)$ is chosen so that $f\left(y_{t}\right) \sin (t)$ satisfies the hypothesis $\left(\mathrm{H}_{2}\right)$, $\left(\mathrm{H}_{3}\right)$. Since the characteristic equation for (6.3) has no imaginary roots, equation (6.3) has no nonzero periodic solutions. Therefore, Corollary (3.3) applies here and yields a $2 \pi$-periodic solution for the boundary value problem $(6.5),(6.6)$. 
Next we consider examples with regard to the degenerate functional differential equations discussed earlier in $\$ 4$. We want first to illustrate the difference between the invertibility of $M+N X_{\Phi}$ as an operator on $R^{n}$ for each $\theta \in[a-h, a]$ and that of $M+N X$ as an operator on $C_{h}$. Consider the following two dimensional system

$$
\begin{aligned}
& u^{\prime}(t)=f_{1}\left(t, u_{t}, v_{t}\right), \\
& v^{\prime}(t)=v(t)+f_{2}\left(t, u_{t}, v_{t}\right), \quad t \in[0,2],
\end{aligned}
$$

subject to the boundary condition (1.2) with boundary operators

$$
M=\left(\begin{array}{ll}
1 & 0 \\
0 & 0
\end{array}\right), \quad N=\left(\begin{array}{ll}
0 & 0 \\
0 & 1
\end{array}\right)
$$

The fundamental solution matrix for the reduced linear equation is

$$
\Phi(t)=\left(\begin{array}{ll}
1 & 0 \\
0 & e^{t}
\end{array}\right)
$$

The matrix $\Gamma(\theta)$ of Theorem 4.2 is given by the inverse of

$$
M+N \Phi(2-\theta)=\left(\begin{array}{cc}
1 & 0 \\
0 & e^{2-\theta}
\end{array}\right)
$$

which is clearly invertible for every $\theta$. However the operator $M+N X$ when applied to $q=\left(q_{1}, q_{2}\right) \in C_{h}$, say $h=1$, yields

$$
(M+N X) q=\left(\begin{array}{c}
q_{1}(0) \\
q_{2}(0) e^{2-\theta}
\end{array}\right),
$$

which shows that it is not one-to-one (both functions $(0,0)$ and $(t, t)$ are in $C_{h}$ and mapped into $(0,0)$ in $\left.C_{h}\right)$. This shows that condition (4.10) cannot be eliminated.

We now wish to show that Theorem (4.2) applies to certain equations with periodic boundary conditions. Consider the one dimensional equation

$$
y^{\prime}(t)=-y(t)+f\left(t, y_{t}\right), \quad t \geqq 0,
$$

where $f\left(t+\pi, y_{t}\right)=f\left(t, y_{t}\right)$ for all $t \geqq 0$ and satisfies $\left(\mathrm{H}_{2}\right),\left(\mathrm{H}_{3}\right)$, together with the periodic boundary conditions

$$
y_{0}=y_{\pi}, \quad y_{0}, y_{\pi} \in C[-1,0] .
$$

It is easy to see that in this case, $\Gamma(\theta)=\left(1-e^{-\pi-\theta}\right)^{-1}$ which satisfies condition (4.10), i.e.

$$
\left\|\Gamma N X_{\Phi}\right\|=\sup _{-1 \leqq \theta \leqq 0}\left|\frac{e^{-\pi-\theta}}{\left(1-e^{-\pi-\theta}\right)}\right| \leqq \frac{1}{e^{\pi-1}-1}<1 .
$$

Thus, Theorem (4.2) applies here and yields a solution to (6.7), (6.8).

Finally, we discuss an example involving integral boundary conditions which illustrates the improvement made through Theorem (4.1) over the previous results [8]. Consider

$$
y^{\prime}(t)=f\left(t, y_{t}\right), \quad M y_{a}+N y_{b}=0, \quad y_{a}, y_{b} \in C_{h},
$$


where $M q(\theta)=q(\theta)-\frac{1}{4} \bar{q}(a), N q(\theta)=\frac{1}{4} \bar{q}(a)+\frac{1}{4} \int_{\theta}^{a} q(\tau) d \tau, \theta \in[a-h, a] ; h<b-a$, and $\bar{q}(a)$ is the constant function in $C_{h}$ with value $q(a)$. It is easy to see that $M+N$ $=I+\frac{1}{4} A, A q(\theta)=\int_{\theta}^{a} q(\tau) d \tau$. If $h=1$, then $\|A\|_{a}<1$. Thus, $(M+N)^{-1}$ exists and satisfies $\left\|(M+N)^{-1}\right\|_{a} \leqq \frac{4}{3}$. Moreover, we have $\|N\|_{a}<\frac{3}{4}$. Therefore $\left\|(M+N)^{-1} N\right\|_{a}$ $\leqq\left\|(M+N)^{-1}\right\|_{a}\|N\|_{a}<1$, and Theorem (4.1) applies. On the other hand, no theorem in [8] would apply in this case.

\section{REFERENCES}

1. K. L. Cooke, Some recent work on functional-differential equations, Proc. U.S.-Japan Seminar on Differential and Functional Equations (Minneapolis, Minn., 1967), Benjamin, New York, 1967, pp. 27-47. MR 36 \#5462.

2. R. D. Driver, Existence and stability of solutions of a delay-differential system, Arch. Rational Mech. Anal. 10 (1962), 401-426. MR 25 \#5260.

3. W. Dubrovskiī, Sur certaines équations intégrales nonlinéaires, Uč. Zap. Moskov. Gos. Univ. Mat. 30 (1939), 49-60.

4. J. Dugundji, An extension of Tietze's theorem, Pacific J. Math. 1 (1951), 353-367. MR 13, 373.

5. N. Dunford and J. T. Schwartz, Linear operators. I: General theory, Pure and Appl. Math., vol. 7, Interscience, New York, 1958. MR 22 \#8302.

6. R. E. Edwards, Functional analysis. Theory and applications, Holt, Rinehart and Winston, New York, 1965. MR 36 \#4308.

7. R. E. Fennell, Periodic solutions of functional differential equations, J. Math. Anal. Appl. (to appear).

8. R. Fennell and P. Waltman, A boundary value problem for a system of nonlinear functional differential equations, J. Math. Anal. Appl. 26 (1969), 447-453. MR 38 \#6185.

9. A. Granas, The theory of compact vector fields and some of its applications to topology and functional spaces. I, Rozprawy Mat. 30 (1962), 93 pp. MR 26 \#6743.

10. A. Halanay, Differential equations: Stability, oscillations, time lags, Academic Press, New York, 1966. MR 35 \#6938.

11. J. K. Hale, Functional differential equations, Lectures, University of California, Los Angeles, 1968-1969.

12. D. Henry, The adjoint linear functional equation and boundary value problems, J. Differential Equations 9 (1971), 55-66.

13. J. Kato, Asymptotic behaviors in functional differential equations, Tôhoku Math. J. (2) 18 (1966), 174-215. MR 34 \#6263.

14. M. Z. Nashed and J. S. W. Wong, Some variants of a fixed point theorem of Krasnoselskii and applications to nonlinear integral equations, J. Math. Mech. 18 (1969), 767-777. MR 38 \#6416.

Courant Institute of Mathematical Sciences, New York University, New York, NEW YORK 10012

Department of Mathematics, University of Iowa, Iowa City, Iowa 52240

Department of Mathematics, Carnegie-Mellon University, Pittsburgh, Pennsylvania 15213

Current address of both authors: Department of Mathematics, University of Iowa, Iowa City, Iowa 52240 